\title{
Come nasce un lettore. Ricordi di lettura e memorie di educazione familiare a partire dal progetto Madeleine in biblioteca
}

\author{
Monica Dati ${ }^{1}$
}

\begin{abstract}
Il presente articolo vuole essere un resoconto del progetto Madeleine in biblioteca, realizzato in collaborazione con la biblioteca civica "Agorà" di Lucca e volto al recupero di storie e memorie di lettura, dall'infanzia all'età adulta. L'attenzione in questa sede viene posta ai primissimi ricordi e al contesto familiare attraverso le testimonianze orali dei partecipanti, utilizzate per costruire uno spazio web dedicato (www.madeleineinbiblioteca.it) e uno specifico seminario sulla lettura di nascosto dove emerge il ruolo dell'autorità dei genitori nel decidere cosa è opportuno o meno leggere. Un percorso per riflettere sull'importanza della lettura e la sua storia e per ricostruire il passato attingendo dalle narrazioni storiche dei non specialisti secondo un approccio di Public History.
\end{abstract}

Parole chiave: storia della lettura, Public History, educazione familiare, autobiografia, memorie familiari.

\begin{abstract}
The paper is focused on the Madeleine in biblioteca project, which has been realized in cooperation with "Agorà" civic library in Lucca (Italy). It was aimed at recovering reading stories, from childhood to adulthood. The attention is here placed on the earliest memories and the family context, through library users' oral testimonies, also useful to achieve a dedicated website (www.madeleineinbiblioteca.it), and a specific workshop on secretly reading and family censorship. A path, this one here shown, to reflect on the importance of reading and its history and to involve the public of non-specialists, in the building of the historical narratives according to a Public History methods.
\end{abstract}

Keywords: history of reading, Public History, family education, autobiography, family memories.

${ }^{1}$ Dottoranda in Scienze della formazione e Psicologia presso il Dipartimento di Formazione, Lingue, Intercultura, Letterature e Psicologia (FORLILPSI) dell'Università degli Studi di Firenze (d'ora in poi, laddove non diversamente specificato, le note a piè di pagina si intendono a cura dell' Autrice, N.d.R.). 
1. Un laboratorio di ricordi e storie di lettura tra autobiografia e memorie familiari

Non so quel che feci sino a cinque o sei anni,
non so come imparai a leggere,
ricordo soltanto le mie prime letture
e l'effetto che produssero su di me:
è il tempo a cui faccio risalire
senza più interruzioni
la coscienza di me stesso.
Jean-Jacques Rousseau
(1782-1789, trad. it. 1956, p. 20)

Il campo di studi relativo alla storia della lettura è estremamente ampio, articolato, diversificato, ricco di fonti documentarie molto eterogenee che ne sottolineano la sua affascinante complessità (Cavallo, Chartier, 1995; Darnton, 1994; Vivarelli, 2018). Tra queste rivestono un ruolo non secondario le testimonianze autobiografiche dei lettori, le loro esperienze intime, uniche e soggettive, le loro storie individuali che partecipi di una storia collettiva possono metterne in luce gli aspetti meno indagati e trascurati, quelli che hanno a che fare con i sentimenti, le emozioni, le spinte ideali. Di queste esperienze oggi si parla poco per quanto la lettura sia una delle pratiche centrali della nostra vita emotiva ed intellettuale e nonostante il libro rimanga tra le fonti di apprendimento principale non solo a livello culturale ma anche sociale, etico e civile (Nobile, 2004). Ogni libro incontrato può contribuire a renderci una persona diversa, modellando la visione di noi stessi e del mondo, proprio come evidenzia Primo Levi: «Quanto delle nostre radici viene dai libri che abbiamo letti? Tutto poco o niente a seconda dell'ambiente in cui siamo nati, della temperatura del nostro sangue, del labirinto che la sorte ci ha assegnato. Non c'è una regola» (1981, p. 19).

Da qui l'idea di mettersi in ascolto e di prestare attenzione alle parole dei lettori così come suggerito da Michèle Petit (1993) che da decenni intervista bambini, adolescenti, uomini e donne di tutte le estrazioni sociali, di campagne, quartieri popolari e circoli di lettura e da Alberto Manguel che in suo famoso volume afferma: «la storia della lettura è la storia di ciascun lettore» (1997, trad. it. 2010, p. 324). Con questa semplice affermazione, l'autore argentino vuole sottolineare come tante piccole storie di apprendimento informale vadano a comporre in realtà un quadro più grande, una galleria che incede di pari passo con la Storia 
e parla di promozione sociale, banchi di scuola, censure, biblioteche, genitori e nonni, di libri imposti, amati ed odiati, letti di nascosto.

$\grave{E}$ in questo contesto, brevemente delineato nelle sue caratteristiche principali, che si colloca il progetto Madeleine in biblioteca che ha visto gli utenti della biblioteca civica di Lucca donare storie e racconti da restituire alla collettività per promuovere la riflessione individuale e collettiva lavorando in una direzione di Public History, approccio generato innanzitutto dal desiderio del pubblico di prendere parte alla documentazione, scrittura e discussione sul passato (Cauvin, 2016; Frisch, 1990):

La tipica demarche della Public History prevede infatti un forte coinvolgimento delle persone, delle collettività, e di tutto il loro patrimonio di ricordi, immagini, oggetti. Un universo di significati e di esperienze, cognitive e affettive, che può essere esplorato in molti modi. $\mathrm{E}$ in molti modi possono essere creati dei legami, delle contaminazioni e anche delle vere e proprie co-costruzioni dei significati storici. Renderci conto di questi aspetti significa offrire a molte persone, ma in particolare a tutti coloro che vivono all' interno di contesti educativi formali, la possibilità di utilizzare questo grande patrimonio, di contribuire ad arricchirlo e comunicarlo (Bandini, 2020, p. 48 ).

Il progetto è stato realizzato nei mesi di marzo-giugno 2020 tramite interviste audioregistrate sviluppate integrando un questionario utilizzato da Piero Innocenti negli anni Ottanta ${ }^{2}$. Gli utenti della biblioteca hanno così risposto a domande relative ai loro primi ricordi di lettura e al contesto familiare, alle opere lette ed imposte a scuola, al loro rapporto con i libri dall'infanzia all'età adulta. Sono state raccolte quaranta testimonianze provenienti da profili diversi per età, genere, titolo di studio e professione che hanno messo in evidenza l'importanza della lettura e il ruolo fondamentale che può avere nel corso di un'esistenza. Insegnanti, lavoratori, pensionati, studenti, accomunati dall'amore per i libri, hanno donato i loro ricordi e memorie mostrando quanto sia fondamentale leggere per la crescita personale, il piacere che ne deriva, il suo porsi come

${ }^{2} \mathrm{P}$. Innocenti (1989): La pratica del leggere: con ottanta interviste a lettori per vocazione, per mestiere, per sensualità, per inedia (Vol. 4). Milano: Editrice bibliografica. Si elencano alcune delle domande. Qual è il tuo primo ricordo di lettura? Cosa hai letto durante l'infanzia? I genitori e la famiglia che ruolo hanno rivestito? Che ricordi hai della lettura a scuola? Alle elementari, medie ed eventualmente alle scuole superiori? C'è un ricordo particolare della tua vita legato ad un libro? Che rapporto hai con le biblioteche? Ci sono libri che hai odiato? Il tuo libro preferito? Hai mai letto di nascosto? Perché? Che cosa significa per te leggere? 
leva di cambiamento e strumento di comprensione della realtà. Con quali modalità far uscire queste importanti testimonianze dal cassetto?

La Rete è sembrata in una prima fase lo spazio più adatto per rendere effettivo lo scambio e il coinvolgimento dei soggetti e per garantire un'efficace restituzione della memoria. Terminate le interviste si è pertanto proceduto all'ideazione e progettazione di un sito web (www. madeleineinbiblioteca.it), collegato a una pagina Instagram per allargare il bacino di partecipazione e condivisione digitale dell'esperienza e avvalersi delle potenzialità offerte dalla Storia digitale. Quest'ultima si rivela una prospettiva particolarmente congeniale agli studi sulle memorie educative soprattutto nel favorire un legame tra la ricerca accademica, $\mathrm{i}$ luoghi di apprendimento ed i contesti di vita. Un approccio che si avvale delle nuove tecnologie di comunicazione aiutando a dare parola non solo ai singoli ma anche ad associazioni e gruppi, utilissimo ad incorporare storie dal basso nella narrazione storica con modalità non sempre possibili nella stampa, nelle esposizioni e nei manuali scolastici.

In un secondo momento si è deciso di procedere all'organizzazione di un seminario, costruito avvalendosi delle testimonianze raccolte, per favorire una più incisiva riflessione collettiva sul racconto della storia di vita ma soprattutto un collegamento con il territorio e la comunità cittadina. Un laboratorio inteso come momento di dialogo tra i soggetti nel sostegno alla contestualizzazione della memoria storica e come spazio per un dibattito comune volto a superare la visione dualistica del dare e ricevere educazione, dell'insegnare e dello studiare. La scelta è ricaduta sul tema della lettura di nascosto, argomento ritenuto particolarmente originale e concordato insieme alla biblioteca civica che ogni anno partecipa all'iniziativa promossa da Aib (Associazione italiana biblioteche) in occasione della giornata dedicata ai libri bruciati nei roghi nazisti ${ }^{3}$.

Il progetto Madeleine ha permesso dunque di approfondire diversi aspetti legati alla lettura e si è rivelato un'opportunità per indagare anche il ruolo della famiglia nella sua promozione evidenziando contestualmente il contributo fondamentale della fonti orali e autobiografiche nella ricostruzione della storia di educazione familiare. «Presenti o assenti, premurosi o opprimenti, dobbiamo fare i conti con loro: i genitori. Non esiste autobiografia che non dedichi almeno una parte del racconto al rapporto con i genitori e alle storie di famiglia che immancabilmente

${ }^{3} \mathrm{Si}$ veda https://www.aib.it/attivita/2020/80761-libri-salvati-letture-2020/ (data di ultima consultazione: 12.2.21). 
raccontano la storia dell'auto-biografo» (Benelli, 2008, p. 127). L'autobiografia dunque,

non è un atto puramente autoreferenziale, implica necessariamente l'incontro con altre storie che si intrecciano a quella dello scrivente. Se l'autobiografia è la ricostruzione di un'esistenza, il ruolo della famiglia e, nella fattispecie dei genitori, è il punto di partenza per comprendere certe scelte e le conseguenze di un tipo di educazione sul vissuto delle persone (Ibidem).

Come sottolineato da Macinai e Oliviero (2017), la storia dell'educazione familiare è rimasta per lungo tempo implicita alla più generale storia della famiglia o a quella dell'infanzia, a cominciare da alcuni "grandi classici”, come il testo di Philippe Ariès (1960) e gli studi di Egle Becchi (1996); lavori di taglio più specificatamente sociologico come quelli di Chiara Saraceno (1998) e di Marzio Barbagli (2003); o, ancora, studi storico-sociali, come quello di Maria Casalini (2010) sulla storia delle famiglie comuniste italiane, dove traspaiono rimandi di carattere educativo.

Si tratta però di alcuni esempi «all'interno della più generale storia della famiglia, che vanta ormai una solida tradizione storiografica, influenzata e rinnovata da molteplici prospettive di ricerca come gli studi di genere» (Macinai, Oliviero, 2017, p. 8) e da nuove metodologie fornite dagli approcci quantitativi. Decidere di raccogliere testimonianze autobiografiche e ascoltare ricordi di lettura, e di analizzare le esperienze in cui essa ha giocato un ruolo essenziale, può pertanto offrire una prospettiva diversa in grado di capire come la storia è entrata nella vita delle persone (Portelli, 2007), indagando in profondità aspetti destinati a rimanere silenti (la pienezza delle emozioni, le difficoltà quotidiane e personali, le conquiste e i traguardi, il ruolo dell'educazione familiare nell'incentivare o meno questo fondamentale apprendimento):

la casa abitata anche dai libri, molti, pochi, irrilevanti è un altro destino familiare. Se si è imparato da piccoli a sfogliarli con qualcuno, scarabocchiarli, a strapazzarli al di là delle reazioni comprensive o furiose dei grandi, contrassegnano la nostra storia più di ogni suppellettile. I libri che abbiamo visto tra le mani di fratelli o sorelle maggiori, dei genitori, la sera tardi, commentati e discussi, ci hanno proiettato oltre le pareti, nell'immaginario e nel bisogno di entrare nelle vite altrui. Nelle altre case e nei paesaggi, nelle avventure umane e impossibili. I ricordi di famiglia di chi ha imparato a considerare i libri degli abitatori silenziosi delle stanze sono diversi da quelli di chi ne ha appreso l'esistenza al di fuori della casa. Di chi non è mai stato accompagnato in una libreria, per sceglierli, guardarli soltanto, desiderarli tutti (Demetrio, 2002, p. 124). 
Di questi aspetti si occupano i successivi paragrafi, dedicati rispettivamente al ruolo dei genitori emerso dalle interviste e alla censura familiare: argomento, quest'ultimo, trattato nel seminario sulla "lettura di nascosto" dal titolo Libri clandestini: letti di nascosto, censurati e proibiti. Un excursus storico. Nell'esporre le due esperienze si farà spesso riferimento alle testimonianze raccolte, ma anche ad autobiografie letterarie, utilizzate nel seminario come esempi per stimolare ricordi ed esperienze, creare momenti di riflessione e di confronto tra soggetti (Benelli, 2020; Cambi, 2005):

nelle attività di Public History, infatti, possiamo individuare sia la produzione di autobiografie sia l'organizzazione di attività di lettura, elaborazione e commento delle autobiografie, rivolte ad un pubblico specifico. La costruzione della fonte storica, in questo modo, entra a far parte, fin da subito di un circuito di partecipazione comunitaria (Bandini, 2020, p. 49).

\title{
2. Qual è il tuo primo ricordo di lettura? Genitori e figli attorno al libro ${ }^{4}$
}

\author{
Allora ero geloso di mia madre \\ e decisi di soffiarle le parole. \\ Jean Paul Sartre \\ (1963, trad. it. 1982, p. 36) \\ L'amore che io avevo per i libri \\ mi era stato instillato \\ nell'animo dal caro \\ e benedetto padre mio. \\ Luigi Settembrini
} (1961, p.10)

Qual è il tuo primo ricordo di lettura? Si tratta di una delle tante domande rivolte agli utenti della biblioteca civica "Agorà" che hanno partecipato al progetto Madeleine in biblioteca donando le loro memorie e i loro preziosi ricordi. Un quesito carico di significato perché, come ricorda Franco Cambi, il libro

è sempre un'esperienza aperta sul mondo, una "finestra" che ci presenta la sua varietà, la sua ricchezza, la sua pregnanza per noi e, così facendo, svi-

${ }^{4}$ Il titolo fa riferimento a un articolo di F. Cambi (2012): Genitori e figli intorno ad un libro. Rivista Italiana di Educazione Familiare, n. 2, pp. 23-27. 
luppa la nostra sensibilità, la rende più variegata e più fine. Sempre, in ogni età della vita. E qui sta la forza della lettura. Che va coltivata con precisione e decisione. Essa è un ponte tra noi e la realtà, in un rapporto intensamente dinamico. Così anche la lettura è un'arte che va appresa (e coltivata) presto. Ci sarà compagna per tutta la vita, crescerà con noi e noi cresceremo con lei (Cambi, 2012, p. 23).

Le risposte dei lettori hanno messo ben in evidenza l'importanza delle agenzie educative della prima infanzia, ci riferiamo in particolare al ruolo dei genitori: sono loro a organizzare la vita quotidiana dei figli, a farli crescere, a soddisfarne i bisogni, a curare la rete delle esperienze più significative, compresa la lettura.

Come impossessarsi della "magia" del libro e del piacere di leggere già dall'età prescolare? Sartre «affascinato dal mondo dei libri che lo circonda, è assalito dalla curiosità di impadronirsi del loro misterioso contenuto» (Nobile, 2004, p. 141), benché ancora non capace di una comprensione autonoma. Ne sottrae uno e si rifugia in un ripostiglio, dove lo sfoglia per capirne i quasi esoterici poteri. Scoperto dai genitori, questi convengono che è giunto il momento di insegnargli a leggere: «ero pazzo di felicità, mie quelle voci che mio nonno rianimava con il suo sguardo, che egli capiva e io non capivo. Mi lasciarono vagabondare tra i libri e diedi l'assalto all'umano sapere. È stato questo a formarmi» (Sartre, 1963, trad. it. 1982, p. 33).

Come per Sartre, in molte delle interviste raccolte il primo ricordo di lettura coincide con il genitore che legge ad alta voce, con l'ascolto di storie, racconti e favole. Di seguito alcuni esempi:

Oltre agli immancabili Pinocchio e Gian Burrasca (appuntamenti fissi con le scuole elementari della mia epoca), vorrei scomodare il Gianni Rodari di Venti storie più una (serie di racconti fantastici fra fiaba e tradizione popolare) che mi leggeva mia madre prima di andare a letto. Ancora oggi mi ricordo certi passaggi di quei racconti, a dir poco suggestivi, e credo che molto di quello che sono divenuto poi sia scaturito proprio da quelle visioni, rimaste indelebili nella mia mente (Marco, 1978).

Nella mia memoria, il primo libro che ricordo di aver ascoltato - a puntate - dalla voce di mia zia Elena è Il giardino segreto della Burnett: avrò avuto circa cinque anni e l'idea di un parco nascosto dietro ad un muro ricoperto di fitta vegetazione solleticava la mia immaginazione e mi spingeva a chiedere di andare sempre più avanti con la storia, per sapere se la bambina sarebbe o meno riuscita ad accedervi (Cristiana, 1980). 
I primi libri che ricordo sono sicuramente quelli di fiabe e filastrocche che i miei leggevano a me e mia sorella per farci addormentare o semplicemente passare il tempo. Alle scuole elementari inizia il mio vero amore per la lettura. Forse il primo libro di cui ho ricordo è L'incompreso, me lo leggeva mia mamma e alla fine, ricordo, piangevamo entrambe (Maria Chiara, 1982).

Sono giorni che ci penso, credo che sia mia mamma che mi legge Storia di una gabbianella e del gatto che le insegnò a volare di Sepúlveda. Sì, perché dovevo farci qualcosa con la scuola, ma è un ricordo vago, qualcuno che mi legge qualcosa (Elena, 1996).

Nel mondo occidentale l'abitudine di leggere ai bambini ha una lunga tradizione, come ricordato da Alberto Manguel, che nel volume Una storia della lettura cita vari esempi, attingendo dall'iconografia cristiana e riportando un carteggio del 1399 dove il notaio toscano Ser Lapo Mazzei, scrive all'amico Francesco di Marco Datini chiedendogli in prestito I fioretti di San Francesco per leggerli ai suoi figli: «I bambini ne traggono diletto nelle sere d'inverno perché come tu ben sai è di facile lettura» (Manguel, 1997, trad. it. 2010, p. 126).

Negli ultimi trent'anni su questo comportamento sono convogliate numerose attenzioni per incentivarlo in maniera strutturata: in Europa si costituiscono, per esempio, «l'associazione francese L.I.R.E. (Bergeron, 2009), il programma nazionale britannico Bookstart (Moore, Wade, 2003), l'italiana Nati per Leggere, che dal 2009 sostiene e incoraggia le pratiche di lettura ad alta voce fin dalla nascita (Manetti, 2006)» (Scotti, 2017, p. 42).

Anche Bianca Pitzorno, tra le più grandi scrittrici per l'infanzia, nel suo Storia delle mie storie afferma che il piacere della lettura viene appreso grazie all'esempio degli adulti che leggono, e descrive come il suo amore per i libri sia nato proprio per merito della pratica dell'ascolto. Come tutti i bambini, è stata circondata da storie anche prima di essere in grado di leggere:

Mia madre all'ora dei pasti prese l'abitudine a raccontarci delle fiabe, era bravissima a catturare la nostra attenzione creando un clima di grande aspettativa e quando arrivava al punto culminante senza parere avvicinava il cucchiaio pieno a una delle sue vittime. Mio fratello, incantato spalancava la bocca e giù la minestrina o gli spinaci! Io invece ascoltavo ma vigile; spalancavo gli occhi ma stringevo le labbra con i denti e non c'era verso di farmi aprire la bocca a nessun costo. Però ascoltavo ma di quelle storie stranamente non mi è rimasto alcun ricordo. Così come non ricordo nessuna delle fiabe classiche che certamente 
mi furono raccontate negli anni successivi. Dico certamente perché so che a otto-nove anni quando sentivo nominare Cenerentola, Barbablì, Il Gatto con gli stivali o la Bella addormentata sapevo di chi si stava parlando (1995, p. 47).

I ricordi delle prime esperienze di ascolto di storie ricorrono spesso nelle biografie o autobiografie di scrittori e poeti. Ecco come lo racconta una scrittrice americana, Eudora Welty:

fin da quando avevo più di due o tre anni ho imparato che ogni stanza della nostra casa, a qualsiasi ora del giorno, era lì perché qualcuno leggesse o ascoltasse qualcuno leggere. A me leggeva la mamma. Probabilmente non le davo tregua e a volte la mamma mi leggeva in cucina, mentre sbatteva il burro nella zangola e il battito regolare dello stantuffo accompagnava ogni storia (1983, trad. it. 2011, p. 66).

Nelle testimonianze delle due scrittrici ritroviamo la figura centrale della madre come prima educatrice, tradizionalmente associata all'impegno lavorativo casalingo. Nel tempo però i costumi sono cambiati e in alcune delle interviste si intravede, seppur timidamente, anche un non scontato ruolo paterno. Si tratta di una dimensione ancora poco indagata ma recenti indagini internazionali sottolineano l'importanza della figura paterna nella promozione della lettura (Clark, 2012; Scotti, 2017) insieme a studi volti ad analizzare i cambiamenti culturali e sociali del ruolo dei padri in atto nella società (Miniati, 2018; Stramaglia, 2008; Zoja, 2000).

Il primo ricordo legato alla lettura è l'immagine di mio padre che mi legge le fiabe classiche dei fratelli Grimm. Uno dei libri che mi ha letto e che riaffiora subito alla mente è stato Piccole donne (Giulia, 1986).

Eravamo alle elementari e ci avevano portato a vedere una mostra di libri da comprare e io ero rimasto colpito da un libro sull'Egitto, una cosa carina perché mio padre era un grande appassionato, e c'era stata questa unione di intenti, non ricordo quanto l'ho pagato e dove sia. Un libro sulla storia egiziana molto semplice, per le elementari, con molte figure e poche pagine. Questo è il mio primo ricordo (Fabio, 1982).

Anche a casa, mio papà nel Dopoguerra comprava La Domenica del Corriere, La tribuna illustrata, ce li passavamo, ed era quasi un gruppo di lettura casalingo (Marusca, 1938).

Anche Elias Canetti riconosce al padre di avergli insegnato il piacere di leggere: «mio padre mi portò un libro, The Arabian Nights, in un'edi- 
zione adatta alla mia età (...). Mi lesse ad alta voce una storia: altrettanto belle sarebbero state tutte le altre. Dovevo cercare di leggerle da solo e poi la sera raccontargliele. Finito il libro me ne avrebbe portato un altro» (1977, trad. it. 1980, p. 59).

Un autore di libri per bambini come Bruno Tognolini nel volume Leggimi Forte narra e commenta la sua esperienza di decennale lettura alla figlia spiegando come i libri possano unire profondamente adulti e bambini, creando un rapporto esclusivo di curiosità, condivisione e complicità anche per i padri:

il racconto della cena ripetuto ogni sera uguale, quando mia figlia era molto piccola, per farla mangiare, per una stagione fu il racconto dell'origine del mondo secondo Tolkien, tratto dal Silmarillion o una sua semplificazione favolosa; per un'altra stagione fu una versione adattata, drasticamente ridotta alla lunghezza di una pappa, della Storia infinita di Ende (2006, p. 98).

Si tratta di un momento di intimità padre-figlia lontanissimo dalla figura paterna che incute timore, assente e avulsa dai compiti di accudimento che ricorre spesso all'interno di testimonianze e casi letterari del secondo Novecento, da Franz Kafka a Montale, da De Chirico a Guttuso (Miniati, 2018), ma anche in alcune autobiografie presenti presso l'archivio diaristico di Pieve Santo Stefano (Ar). Un emblematico esempio è rappresentato dal diario Come tu mi vuoi di Flora Ritter: giovane di famiglia borghese, nel pieno del movimento politico del Sessantotto, scrive di come il severo padre non le conceda alcuna libertà e le vieti addirittura di leggere «l'immorale Tolstoj» (Archivio Diaristico Nazionale, Collocazione DP/01). Un ricordo di controllo familiare che ci permette di introdurre l'argomento del prossimo paragrafo relativo alla censura e alla lettura di nascosto, oggetto del già citato seminario collegato al progetto Madeleine costruito con le testimonianze orali raccolte: un momento di riflessione collettiva e di restituzione alla comunità delle memorie donate dagli utenti della biblioteca.

\section{Sotto controllo: libri letti di nascosto e censura familiare}

Mi venivano dati soltanto libri per l'infanzia scelti con cautela; libri che ammettevano le stesse verità e gli stessi valori dei miei genitori e delle mie insegnanti.

Simone de Beauvoir (1958, trad. it.1994, p. 53) 
Il 10 settembre 2020 presso la biblioteca civica di Lucca si è svolto il seminario Libri Clandestini. Letti di nascosto, censurati, proibiti costruito avvalendosi delle testimonianze raccolte tramite le interviste del progetto Madeleine, con particolare riferimento alle risposte alla domanda: "Hai mai letto di nascosto?". Si è trattato di un momento di dialogo nel sostegno alla contestualizzazione della memoria storica e di uno spazio finalizzato al dibattito comune intorno alla tematica delle letture proibite e della censura. Ci sono infatti libri che non si devono leggere, che sono sconsigliati, che esercitano una cattiva influenza, «che possono distrarre dalle cose serie, che potrebbero mettere in dubbio la fede o spingere verso fantasie peccaminose» (The Book Fools Bunch, 2019, p. 8). Libri considerati inadatti, scabrosi, immorali. Letture troppo leggere, disimpegnate, irrispettose, dissidenti, ideologiche, da non nominare. Le esperienze di lettura clandestina possono essere davvero molteplici, oggi come ieri, a seconda del contesto storico, sociale e culturale, dei vissuti personali. Non meno complesso il nesso tra libri e divieti in un rapporto che si evolve nel tempo, ma che abitualmente caratterizza ogni esigenza di pubblica comunicazione.

Partendo da esperienze uniche e soggettive il laboratorio ha esplorato questo variopinto universo. Nella prima parte si è cercato di contestualizzarlo storicamente e di fare delle autobiografie raccolte materiale per promuovere una riflessione collettiva su alcuni specifici percorsi: dal ruolo della Chiesa alla questione di genere, dalla lettura di nascosto degli schiavi afro-americani ai roghi di libri. Nella seconda parte i partecipanti al seminario sono stati invitati a esporre le loro esperienze, a proporre suggerimenti ed esempi in modo da creare un laboratorio interattivo e di reciproco scambio stimolato anche dalla lettura di autobiografie letterarie. In questa sede ci limiteremo esclusivamente al tema dell'autorità familiare, come scrive Henry Miller infatti: «vi è una cosa che distingue le letture dell'infanzia da quelle fatte in epoche posteriori, ed è la mancanza di scelta. I libri che leggiamo da bambini ci sono stati cacciati in mano. Fortunato il bambino che ha dei genitori avveduti!» (1952, trad. it. 1976, p. 37).

Silvia Blezza Picherle in Formare Lettori, promuovere la lettura ricorda come quella della scelta sia un'illusione. I destinatari della letteratura per l'infanzia sono bambini e ragazzi che difficilmente hanno la possibilità di scegliere le letture in base ai propri interessi, inclinazioni e piaceri.

In Italia nel corso dell'Ottocento e Novecento, genitori, insegnanti, educatori, scrittori e critici letterari hanno proposto ed imposto solo le narrazioni 
ritenute "adatte", sotto il profilo educativo, quindi moralmente coerenti con i valori della famiglia e del contesto socio culturale di appartenenza. "Erano preferite le storie che coltivavano animi gentili, che rendevano migliori e contribuivano ad irrobustire il carattere". Si è pure sostenuto che "il ragazzo non può e non deve scegliere da sé le sue letture finché non abbia un gusto educato e responsabile" (2013, p. 44).

I libri ritenuti trasgressivi venivano tagliati e rimaneggiati come è accaduto ai romanzi di Emilio Salgari considerati diseducativi dal mondo cattolico e dalla censura fascista perché troppo crudi e violenti, poco edificanti e capaci di risvegliare fantasie licenziose e l'eccessiva evasione dalla realtà. Pure Pippi Calzelunghe, moderna e rivoluzionaria come la sua autrice Astrid Lindgren, è stata guardata con diffidenza nell'Italia del secondo Dopoguerra perché si pensava che il suo comportamento libero e anticonformista potesse sovvertire i ruoli di genere e i valori tradizionali (Blezza Picherle, 2013). Basti pensare, come ricorda Blezza Picherle, che ancora negli anni Sessanta alcune case editrici si premuravano di assicurare che $\mathrm{i}$ romanzi proposti fossero seri e moralmente ineccepibili per le bambine e le ragazzine. A tal proposito si riporta quanto emerso dal dibattito su una delle tematiche affrontate durante il seminario, che ha avuto particolare seguito e che riguarda la lettura e il suo rapporto con il genere e l'autorità familiare.

Ricordo che mia nonna diceva che sua mamma quando era sera imparava a leggere scrivere e a fare la sua firma ed era costretta a farlo di nascosto perché suo marito le diceva "cosa perdi tempo in queste cose". C'era una questione di genere legata ad un periodo storico in cui alla donna non era richiesto avere cultura (Jessica, 1974).

Memorie e ricordi come questa testimonianza hanno acceso il dibattito sul tema storico dell'educazione delle bambine e delle donne, ai diversi destini per esse pensati e preparati, individuando anche stereotipi legati alla narrativa, alla stampa femminile e al romanzo rosa, ma soprattutto esclusioni e proibizioni.

In particolare, durante il seminario si è cercato di ricostruire gli interventi di disciplinamento, le strategie di controllo dei contenuti e delle coscienze che, nel tentativo di proteggere le donne dai "pericoli" della lettura, miravano alla salvaguardia dell'ordine sociale (Tippelskirch, 2011). La tutela delle lettrici non è passata infatti solo attraverso la censura della produzione letteraria o versioni purgate di opere riservare al pubblico femminile ma anche e soprattutto attraverso il sistema di per- 
messi e divieti familiari (Ulivieri, 2007). Del resto, come ricorda Alberto Manguel, la suocera di Madame Bovary era convinta che i romanzi intossicassero Emma e convinse suo figlio a sospendere l'abbonamento della moglie ad una biblioteca circolante, con il risultato «di sprofondarla ancor più nelle paludi della noia» (Manguel, 1997, trad. it. 2010, p. 294). L'autorità dei genitori e il ruolo che la famiglia riveste nel decidere cosa è opportuno leggere o no sono stati poi approfonditi in modo più generale attraverso una serie di memorie, come quella di Marirosa, oggi in pensione, ex ricercatrice universitaria:

a 15 anni leggevo Hemingway di nascosto, insieme a D.H. Lawrence autori fieramente disapprovati dalla mia famiglia che non ne aveva una conoscenza diretta ma si uniformava all'opinione della borghesia benpensante (a quelli che hanno oggi quindici anni debbono sembrare situazioni da Medioevo).

In riferimento a questa testimonianza un partecipante ha ricordato di aver letto di nascosto Nanà di Émile Zola e ha incrociato questa memoria con un'analoga rappresentazione letteraria, riportando uno stralcio autobiografico di Anaïs Nin, una delle autrici di riferimento nella letteratura erotica, che così ricorda la libreria del padre:

le ore che passavo lì a leggere quando lui era fuori casa, a leggere sopra una seggiola appoggiata ad un'altra seggiola, in moda da poter raggiungere i libri sugli scaffali più alti, quelli che ci erano proibiti. Fu allora che lessi Zola, senza capire la metà, e passai ore a domandarmi come mai gli amanti presi nell'esplosione di una miniera erano stati ritrovati avvinghiati tanto saldamente, che non si poteva separarli, o perché la donna a cui Montecristo aveva dato un sonnifero, più tardi, si scoprì incinta. Impossibile riempire questi spazi vuoti ma io leggevo (1966, trad. it. 1977, p. 117).

In modo analogo, dal pubblico è stato ricordato anche il romanzo autobiografico di Simone de Beauvoir, Memorie di una ragazza per bene, dove l'autrice si racconta attraverso i libri letti, spesso controllati e proibiti dai genitori:

le mie letture continuavano ad essere controllate con lo stesso rigore che in passato; all'infuori della letteratura destinata all'infanzia, o convenientemente purgata, non mi venivano date che pochissime opere scelte, e in più, spesso, $\mathrm{i}$ miei genitori ne censuravano dei passi (1958,trad. it. 1994, p. 85).

Particolarmente interessante la testimonianza di una lettrice che nel ricordare la sua adolescenza racconta con piacere di come rimase affa- 
scinata dalla collana di Angelica la Marchese degli Angelidi Anne e Serge Golon. Per i genitori si trattava di una lettura non indicata per i contenuti troppo espliciti e licenziosi, decise così di trafugarli poco alla volta dalla biblioteca della nonna: «mi ricordo che gli adulti mi sconsigliarono la lettura di quei libri troppo espliciti per una ragazzina, ma io li trafugai tutti poco alla volta e li portai a casa nella mia camera. E così cominciai a vivere le meravigliose avventure di Angelica».

Difficile negare che la passione per la lettura si alimenti della trasgressione, dell'infrazione a divieti immotivati ed ingiusti o vissuti emotivamente come tali. «Scorrendo le autobiografie, spesso il primo incontro con il libro è ricordato come un avvenimento che ha lasciato tracce profonde, anche perché altrettanto spesso la lettura di quel libro si lega al fascino del proibito» (Genovesi, 1999, p. 75). A tal proposito, merita di essere citata una ricerca svolta da Stefania Buccini dell'Università del Wisconsin (1993) che ha per oggetto le letture clandestine e "furti di libri" nelle biografie di scrittori del Settecento come Antonio Genovesi, Carlo Goldoni e Edward Gibbon, e molti altri. Goldoni rammenta di aver letto "la non castissima" Mandragola grazie a un furto e alla sbadataggine del canonico di casa:

C'etoi la Mandragore de Machiavelli. Je ne la connoissois pas: mais j'en avois entendu parler, et je savois bien que ce n'etoit pas une piece tres chate. Je la divorai a la premiere lecture et je l'ai relue dix fois. Ma mère ne faisot pas attention au lovre que je lisois, car c'etoit un Ecclesiastique qui me l'avoit donne; mais mom pere me surprit un jour dans ma chambre, pendant che je fasois des notes et des reamarques sur la Mandragore: Il la connoissoit: il savoit combien cette piece etoit dangerouse pour un jeune homme dix sept ans; il me gronda amerement, et se brouilla avec ce pauvre Chanoine qui n'avoit que par nonchalance (in Buccini, 1993, p. 32) .

Antonio Genovesi, similmente, ricorda così il suo rapporto con i libri proibiti:

La Cleopatra, La Cassandra ed alcuni romanzacci spagnoli furono divorati in poco tempo letti. Quanto più leggeva di queste inette favole più ne diveniva voglioso. Io correa alla mia ruina. I studi filosofici mi parevano insipidi. Io mi ero rallentato nello studio. Mio padre, che se n'era accorto, spiò la cagione e la ritrovò. Egli era severissimo e me ne castigò forse più di quello che io meritava ([1748-1755], 1962, pp. 9-10)

${ }^{5} \mathrm{Il}$ passaggio in oggetto è tratto da Buccini dalle Mémoires di Carlo Goldoni, N.d.R. 
In generale, si è trattato di un seminario-laboratorio molto corposo che ha toccato un ventaglio enorme di temi e che è "nato consapevole" di trascurarne molti altri. Come si evince da alcuni dei ricordi riportati e dagli autori menzionati, la letteratura ritenuta immorale e responsabile di fomentare dissolutezza riveste un ruolo di rilievo nella storia della lettura "clandestina" e delle censure familiari.

Alcuni partecipanti hanno sottolineato anche l'imbarazzo o la difficoltà nel fare letture considerate troppo leggere e frivole. Una lettrice (1956) ricorda: «in casa mia i fumetti erano vietati perché considerati sub-cultura. Io ero attratta da Asterix, che occasionalmente mi veniva consentito. Ho sempre letto molto, per me la letteratura è stata una risorsa essenziale». Elena (1996) nella sua intervista ricorda di essere stata costretta a leggere Harry Potter in determinate fasce orarie: «Ho letto di nascosto ma non un libro in particolare, mi ricordo alle medie e ad inizio superiori i miei non volevano leggessi troppo per diletto per non sottrarre tempo allo studio. Harry Potter e anche altri libri erano permessi solo in certe fasce orarie perché erano ritenuti mero svago».

Due ulteriori emblematici esempi, quelli appena riportati, che sottolineano l'importanza delle fonti autobiografiche nelle ricostruzioni storico- educative, compresa la storia di apprendimenti tanto importanti come la lettura nella cui promozione giocano un ruolo centrale anche famiglia e genitori.

\section{Conclusioni}

Il progetto Madeleine vuole essere un primo tentativo per dimostrare come sia possibile attraverso la restituzione di memorie e ricordi costruire un percorso di riflessione collettiva intorno alla lettura, alle sue molteplici valenze formative e ai variopinti contesti in cui si esercita, non ultimo quello familiare. Una preziosa occasione per applicare i principi della Public History a un terreno inesplorato come la storia della lettura e del libro.

Mettere in pratica un dialogo tra interlocutori diversi, genitori, figli, bibliotecari e ricercatori per stabilire una conoscenza diffusa e condivisa del passato, capace altresì di valorizzare la storia e l'importanza di pensare storicamente, ritenuto un pensare utile per tutta la collettività, incluso il mondo degli apprendimenti informali e i luoghi dove essi si realizzano. Come precedentemente sottolineato, quando si ha a che fare con le storie di vita si affronta inevitabilmente anche il tema dei rapporti 
familiari e dell'educazione ricevuta. Indagare ricordi e memorie di lettura può rappresentare così una modalità inedita ed originale di affrontare storicamente la funzione educativa della famiglia e l'avvio di un possibile percorso di approfondimento che preveda un forte coinvolgimento delle persone e del loro patrimonio di ricordi ed esperienze:

restiamo convinti della significatività della ricerca storiografica sulle memorie educative e della rilevanza che le fonti autobiografiche mantengono per la ricostruzione dei processi di trasformazione delle identità individuali e collettive. Il discorso è più che aperto e corrisponde alla necessità di raccogliere ulteriori elementi di consolidamento per la delineazione di filoni di ricerca, forse ancora poco valorizzati ma ampiamente accreditati sul piano epistemologico, capaci di guardare alle famiglie e all'universo delle relazioni intergenerazionali attraverso una prospettiva bottom-up (Macinai, Oliviero, 2017, p. 18).

\section{Ringraziamenti}

Le biblioteche possono svolgere un ruolo centrale nel collegare mondo accademico e comunità, presentandosi non solo come luoghi dove raccogliere e conservare libri ma centri di esperienza e conoscenza al servizio del pubblico. Alla biblioteca civica "Agorà" di Lucca, uno spazio libero e aperto a una pluralità di soggetti e iniziative, vanno i nostri ringraziamenti per aver accolto questa ricerca e questo progetto.

\section{Riferimenti bibliografici}

Alexandrian S., Luoni B. (1990): Storia della letteratura erotica. Milano: Rusconi.

Ariès Ph. (1960): Padri e figli nell'Europa medievale e moderna. Trad. it. Bari: Laterza, 1968.

Asquer E., Casalini M., Di Biagio A., Ginsborg P. (a cura di) (2010): Famiglie del Novecento. Conflitti, culture e relazioni. Roma: Carocci.

Bandini G., Oliviero S. (a cura di) (2019): Public History of Education. Riflessioni, testimonianze, esperienze. Firenze: Firenze University Press.

Barbagli M., Castiglioni M., Dalla Zuanna G. (2003): Fare famiglia in Italia: un secolo di cambiamenti. Bologna: il Mulino.

Barbagli M., Kertzer D.I.U. (a cura di) (2005): Storia della famiglia in Europa. 3 voll. Roma-Bari: Laterza.

Batini F., Bandini G., Benelli C. (2020): Autobiografia ed educazione. Autobiografie. Ricerche, pratiche, esperienze, n. 1, pp. 47-58. 
Beauvoir S., de (1958): Memorie di una ragazza per bene. Trad. it. Torino: Einaudi, 1994.

Becchi E., Julia D. (a cura di) (1996): Storia dell'infanzia, 2 voll. Roma-Bari: Laterza.

Benelli C. (2008): Le scritture di sé negli Archivi Autobiografici: i ricordi di famiglia. RIEF-Rivista Italiana di Educazione Familiare, n. 1, pp. 127-135.

Bergeron E. (2009): Expériences de lecture à L.I.R.E. à Paris. Toulouse: ERES.

Blezza Picherle S. (2013): Formare lettori, promuovere la lettura: riflessioni e itinerari narrativi tra territorio e scuola. Milano: FrancoAngeli.

Buccini F. (1993): Letture clandestine nelle autobiografie del Settecento. Yearbook of Italian Studies. v. 10, pp. 29-39.

Cambi F. (2005): L'autobiografia come metodo formativo. Roma: Laterza.

Cambi F. (2012): Genitori e figli intorno ad un libro. RIEF-Rivista Italiana di Educazione Familiare, n. 2, pp. 23-27.

Canetti E. (1977): La lingua salvata. Trad. it. Milano: Adelphi, 1980.

Cantatore L. (a cura di) (2017): Primo: leggere. Per un'educazione alla lettura. Roma: Edizioni Conoscenza.

Casalini M. (2010): Famiglie comuniste. Ideologia e vita quotidiana nell'Italia degli anni Cinquanta. Bologna: il Mulino.

Cauvin T. (2016): Public History: A Textbook of Practice. New York and London: Routledge.

Cavallo G., Chartier R. (1995): Storia della lettura nel mondo occidentale. RomaBari: Laterza.

Chambers A. (1993): Il piacere di leggere e come non ucciderlo. Casale Monferrato: Sonda, 2006.

Clark C. (2012): Boys' Reading Commission 2012: A Review of Existing Research Conducted to Underpin the Commission. National Literacy Trust. (https:// eric.ed.gov/?id=ED541405; data di ultima consultazione: 11.2.21).

Darnton R. (1989): Il bacio di Lamourette. Trad. it. Milano: Adelphi, 1994.

Demetrio D. (2002): Album di famiglia. Scrivere i ricordi di casa. Milano: Meltemi.

Demetrio D. (2008): Le scritture famigliari tra memoria e diari del presente. RIEF-Rivista Italiana di Educazione Familiare, n. 1, pp. 19-38.

Demetrio D. (2012): Educare è narrare. Milano-Udine: Mimesis.

Delumeau J., Roche D. (2000): Histoire des pères et de la paternité. Paris: Larousse.

De Sanctis F.M., Federighi P. (1981): Pubblico e biblioteca. Roma: Bulzoni Editore.

Detti E. (2002): Il piacere di leggere. Scandicci (Fi): La Nuova Italia.

Fabre G. (1998): L'elenco: censura fascista, editoria e autori ebrei. Torino: Zamorani.

Frisch M. (1990): A Shared Authority: Essays on the Craft and Meaning of Oral and Public History. Albany (NY): State of New York University Press.

Genovesi A. [1748-1755]: Vita di A.G. In Id.: Autobiografia, lettere e altri scritti, a cura di Gennaro Savarese. Milano: Feltrinelli, 1962, pp. 9-10. 
Genovesi G. (1999): L'educazione dei figli: L'Ottocento (vol. 5). Scandicci (Fi): La Nuova Italia.

Ginsborg P. (2014): Famiglia Novecento: vita familiare, rivoluzione e dittature, 1900-1950. Torino: Einaudi.

Ginzburg C. (1976): Il formaggio e i vermi. Torino: Einaudi.

Innocenti P. (1989): La pratica del leggere: con ottanta interviste a lettori per vocazione, per mestiere, per sensualità, per inedia (vol. 4). Milano: Editrice bibliografica.

Ladefroux R., Petit M., Gardien C.M. (1993): Lecteurs en campagne. Les ruraux lisent-ils autrement? Nanterre: BPI.

Levi P. (1981): La ricerca delle radici. Torino: Einaudi.

Lyons M. (2010): A History of Reading and Writing: in the Western World. Basingstoke: Palgrave Macmillan.

Macinai E., Oliviero S. (2017): Le memorie di educazione familiare: voci, autobiografie, suoni e immagini. Prospettive di ricerca. RIEF-Rivista Italiana di Educazione Familiare, n.1, pp. 7-18.

Manetti, S. (2006): Nati per Leggere, un intervento di comunità: a che punto siamo? Quaderni acp, 13(5), pp. 195-198.

Manguel A. (1997): Una storia della lettura. Milano: Feltrinelli, 2010.

Miller H.(1952): I libri della mia vita. Torino: Einaudi, 1976

Miniati L. (2018): Padri di ieri e di oggi. Indagare la genitorialità maschile attraverso la pratica autobiografica. RIEF-Rivista Italiana di Educazione Familiare, pp. 153-165.

Moore M., Wade B. (2003): Bookstart: a Qualitative Evaluation. Educational Review, 55(1), pp. 3-13.

Nin A. (1966): Diario I: 1931-1934. Trad. it. Milano: Bompiani, 1977.

Nobile A. (2004): Lettura e formazione umana. Brescia: La Scuola.

Petit M. (2002): Elogio della lettura. Milano: Ponte alle Grazie, 2010.

Pitzorno B. (1995): Storia delle mie storie. Parma: Pratiche.

Portelli A. (2007): Storie orali: racconto, immaginazione, dialogo. Roma: Donzelli.

Rousseau J.-J. (1782-1789): Confessioni. (Vol. 1). Torino: Utet, 1956

Saraceno C., Naldini M. (1998): Mutamenti della famiglia e politiche sociali in Italia. Bologna: il Mulino.

Sartre J.-P (1963): Le parole. Trad. it. Milano: Il saggiatore, 1982.

Savater F. (1997): A mia madre, mia prima maestra. Il valore di educare. RomaBari: Laterza.

Scotti M. (2017): Leggere ai figli e crescere come padri: uno studio qualitativo sugli effetti della lettura per i padri che leggono ad alta voce. Lifelong Lifewide Learning, 13(29), pp. 42-58.

Settembrini L. (1961): Ricordanze della mia vita e scritti autobiografici. Milano: Feltrinelli.

Stramaglia M. (2008): I nuovi padri. Per una pedagogia della tenerezza. Macerata: EUM. 
The Book Fools Bunch (2019): Il libro dei libri proibiti. Firenze: Edizioni Clichy, 2019.

Tippelskirch X.V. (2011): Sotto controllo: letture femminili in Italia nella prima età moderna. Roma: Viella Libreria Editrice.

Valentino Merletti R., Tognolini B. (2006): Leggimi forte. Milano: Salani.

Ulivieri S. (2007): Educazione al femminile: una storia da scoprire. Milano: Guerini Scientifica.

Violi P. (2020): Breve storia della letteratura rosa. Perugia: Graphe.it.

Vivarelli M. (2018): La lettura: storie, teorie, luoghi. Milano: Editrice bibliografica.

Welty E. (1983): Come sono diventata una scrittrice. Trad. it Roma: Minimum Fax, 2011.

Wolf H. (2006): Storia dell'Indice: il Vaticano e i libri proibiti. Trad. it. Roma: Donzelli, 2006.

Zoja L. (2000): Il gesto di Ettore. Preistoria, storia, attualità e scomparsa del padre. Torino: Bollati Boringhieri.

Riferimenti sitografici

www.aib.it (data di ultima consultazione: 12.2.21)

www.eric.ed.gov (data di ultima consultazione: 11.2.21)

www.madeleineinbiblioteca.it (data di ultima consultazione: 4.2.21) 